\title{
Ramsey-type problems in orientations of graphs *
}

\author{
Bruno Pasqualotto Cavalar ${ }^{1}$ \\ ${ }^{1}$ Instituto de Matemática e Estatística, Universidade de São Paulo \\ Rua do Matão 1010, 05508-090 São Paulo, SP \\ brunopceime.usp.br
}

\begin{abstract}
The Ramsey number $R(H)$ of a graph $H$ is the minimum number $n$ such that there exists a graph $G$ on $n$ vertices with the property that every two-coloring of its edges contains a monochromatic copy of $H$. In this work we study a variant of this notion, called the oriented Ramsey problem, for an acyclic oriented graph $\vec{H}$, in which we require that every orientation $\vec{G}$ of the graph $G$ contains a copy of $\vec{H}$. We also study the threshold function for this problem in random graphs. Finally, we consider the isometric case, in which we require the copy to be isometric, by which we mean that, for every two vertices $x, y \in V(\vec{H})$ and their respective copies $x^{\prime}, y^{\prime}$ in $\vec{G}$, the distance between $x$ and $y$ is equal to the distance between $x^{\prime}$ and $y^{\prime}$.
\end{abstract}

\section{Introduction}

An ordered graph $G$ is a pair $G=\left(G^{\prime},<_{G}\right)$ where $G^{\prime}$ is a graph and $<_{G}$ is a total ordering of the vertices of $G^{\prime}$. For convenience we write $V(G):=V\left(G^{\prime}\right)$ and $E(G):=E\left(G^{\prime}\right)$. When a graph $G$ is equipped with a total ordering of its vertices, we will simply refer to $G$ as an ordered graph without further qualifications. Finally, $G^{\prime}$ is called the underlying unordered graph of $G$.

An ordered graph $G$ is said to contain an ordered graph $H$ if there exists a function $\phi: V(H) \rightarrow V(G)$ such that, for every $x, y \in V(H)$, we have $\phi(x)<_{G} \phi(y)$ if and only if $x<_{H} y$, and $\{i, j\}$ is an edge of $H$ only if $\{\phi(i), \phi(j)\}$ is an edge of $G$. In this case, we call $\phi$ a monotone embedding.

A directed graph or digraph $\vec{G}$ is a pair $\vec{G}=(V, E)$ where $V$ is a set of vertices and $E$ is a set such that $E \subseteq(V \times V) \backslash\{(v, v): v \in V\}$. Just as in the case of undirected graphs, an element of $E$ is called an edge; however, it may also be called an arc to differ from the undirected case. An oriented graph $\vec{G}=(V, E)$ is a digraph where $(u, v) \in E$ implies $(v, u) \notin E$ for every $u, v \in V$. Moreover, an oriented graph $\vec{G}=\left(V_{1}, E_{1}\right)$ is said to be an orientation of a graph $G=\left(V_{2}, E_{2}\right)$ if $V_{1}=V_{2}$ and, for every $u, v \in V_{1}=V_{2}$, we have $\{u, v\} \in E_{2}$ if and only if $(u, v) \in E_{1}$ or $(v, u) \in E_{1}$. In this case, we say that $G$ is the underlying undirected graph of $\vec{G}$. Furthermore, when $\vec{G}$ is an oriented graph, we write $G$ to denote the underlying undirected graph of $\vec{G}$. To avoid confusion, we will always denote a digraph by a capital letter with $\rightarrow$.

Given graphs $H$ and $G$ and an integer $r \geqslant 2$, we write $G \rightarrow(H)_{r}$ if every coloring of the edges of $G$ with $r$ colors contains a monochromatic copy of $H$. When $r=2$, we may simply write $G \rightarrow H$.

\footnotetext{
*Work supported by FAPESP, Proc. 2015/26678-9.
} 
If the graphs $H$ and $G$ are ordered graphs, we write $G \stackrel{\text { ord }}{\longrightarrow} H$ to denote that the monochromatic copy is ordered. The Ramsey number $R(H)$ of a graph $H$ is defined as

$$
R(H):=\inf \left\{n \in \mathbb{N}: K_{n} \rightarrow H\right\} .
$$

When the graph $H$ is an ordered graph, the ordered Ramsey number $R_{<}(H)$ can be defined analogously. Moreover, for convenience we let $R(H)$ denote the Ramsey number of its underlying unordered graph.

Given an oriented graph $\vec{H}$ and a graph $G$, we write $G \rightarrow \vec{H}$ if every orientation of $G$ has an oriented copy of $\vec{H}$. The oriented Ramsey number $\vec{R}(\vec{H})$ is defined as

$$
\vec{R}(\vec{H}):=\inf \left\{n \in \mathbb{N}: K_{n} \rightarrow \vec{H}\right\} .
$$

\section{A bound for the oriented Ramsey number}

To our knowledge, the following is the first bound to appear of the oriented Ramsey number of an oriented graph. Not much has been published since then. Contrary to the traditional Ramsey number, the oriented Ramsey number has been scarcely studied.

Theorem 1 ([Erdôs and Moser 1964]). Let $\vec{K}_{k}$ be the acyclic orientation of $K_{k}$ for some positive integer $k$. We have $2^{(k-1) / 2} \leqslant \vec{R}\left(\vec{K}_{k}\right) \leqslant 2^{k-1}$.

We now give a bound for the oriented Ramsey number of $\vec{H}$ depending on the Ramsey number of $H$. First, we need a bound for the Ramsey number of ordered graphs. Theorem 2 ([Conlon et al. 2017]). There exists a constant $c$ such that, for every ordered graph $H$ on $h$ vertices, we have $R_{<}(H) \leqslant R(H)^{c \log ^{2} h}$.

More precise bounds for $R_{<}(H)$ for specific classes of ordered graphs can be found in [Conlon et al. 2017] and [Balko et al. 2015]. Our first result is as follows. A slightly stronger result is proven in the full paper.

Theorem 3. There exists a constant $c$ such that the following holds. Let $\vec{H}$ be an acyclic oriented graph with $h$ vertices and $H$ its underlying undirected graph. Then

$$
\vec{R}(\vec{H}) \leqslant 2 R(H)^{c \log ^{2} h} .
$$

The proof goes roughly as follows. Let $\vec{F}$ be the oriented graph formed by two disjoint copies of $\vec{H}$, in which one has reversed edges. Let $F$ be the (ordered) underlying undirected graph of $\vec{F}$ equipped with a topological ordering of the vertices. We prove that, if $K_{n} \stackrel{\text { ord }}{\longrightarrow} F$, then $K_{n} \rightarrow \vec{H}$, which implies the result.

\section{An Oriented Ramsey Theorem for Random Graphs}

For a graph $H$, we denote by $m_{2}(H)$ its 2-density, defined as

$$
m_{2}(H):=\max _{F \subseteq H,|V(F)| \geqslant 3} \frac{|E(F)|-1}{|V(F)|-2},
$$

where $F \subseteq H$ means that $F$ is a subgraph of $H$. Let us also denote by $G(n, p)$ the binomial random graph in which each edge appears with probability $p$, independently of each other edge.

The following is a famous result of Rödl and Ruciński [Rödl and Ruciński 1995], which determines, for an undirected graph $H$, the threshold function for $G(n, p) \rightarrow(H)_{r}$. Here we state only the 1-statement. 
Theorem 4 ([Rödl and Ruciński 1995]). Let $r \geqslant 2$ and $H$ be a graph. There exists a constant $C=C(H, r)$ such that, if $p \geqslant C n^{-1 / m_{2}(H)}$, then $\lim _{n \rightarrow \infty} \mathbb{P}\left[G(n, p) \rightarrow(H)_{r}\right]=1$.

We obtained a result for oriented graphs analogous to Theorem 4, described as follows.

Theorem 5. Let $\vec{H}$ be an acyclic oriented graph. There exists a constant $C=C(\vec{H})$ such that, if $p \geqslant C n^{-1 / m_{2}(\vec{H})}$, then

$$
\lim _{n \rightarrow \infty} \mathbb{P}[G(n, p) \rightarrow \vec{H}]=1
$$

Our proof of Theorem 5 adapts the arguments of [Nenadov and Steger 2016], who gave a short proof of Theorem 4 using the method of containers, developed independently by [Balogh et al. 2015] and [Saxton and Thomason 2015]. The technique of using hypergraph containers in random graphs for Ramsey problems was further developed by [Hàn et al. 2016], [Rödl et al. 2016] and [Conlon et al. 2016]. Our approach is also inspired by theirs.

A very rough explanation of the proof idea would be as follows. Applying the hypergraph container lemma of [Balogh et al. 2015] and [Saxton and Thomason 2015], we are able to prove that, if a graph $G$ on $n$ vertices does not satisfy $G \rightarrow \vec{H}$, then there exists a $s$-tuple $\mathcal{T}=\left(\mathcal{T}_{1}, \ldots, \mathcal{T}_{s}\right)$ of subsets of $E(G)$ and a set $\mathcal{C}=\mathcal{C}(\mathcal{T}) \subseteq E\left(K_{n}\right)$ depending only on $\mathcal{T}$ such that

(i) The number $s$ depends only on $\vec{H}$,

(ii) $\bigcup_{i \in[s]} \mathcal{T}_{i} \subseteq E(G) \subseteq \mathcal{C}$,

(iii) $\left|\mathcal{T}_{i}\right|$ is "small" (in a precise technical sense) for every $i \in[s]$,

(iv) $|\mathcal{C}|$ is "small" in a precise technical sense.

Observe that, since $E(G) \subseteq \mathcal{C}(\mathcal{T})$, then $D(\mathcal{T}):=E\left(K_{n}\right) \backslash \mathcal{C}(\mathcal{T})$ satisfies $E(G) \cap$ $D(\mathcal{T})=\emptyset$. Moreover, since $|\mathcal{C}(\mathcal{T})|$ is "small", we have that $|D(\mathcal{T})|$ is "large". Because of this, we can bound the probability of the event $\{G(n, p) \nrightarrow \rightarrow \vec{H}\}$ by the probability that there exists a $s$-tuple $\mathcal{T}$ satisfying (i)-(iii) and such that $G$ avoids $D(\mathcal{T})$. Since the $\mathcal{T}_{i}$ are "small" and $D(\mathcal{T})$ is "large", this probability must also be "small" - that is, we must have $\mathbb{P}[G(n, p) \not \rightarrow \vec{H}]=o(1)$.

\section{The Isometric Oriented Ramsey Number}

For an undirected graph $G$, we denote by $d_{G}(u, v)$ the distance between two vertices $u, v \in V(G)$. Given two oriented graphs $\vec{H}$ and $\vec{F}$, we say that a copy $f: V(\vec{H}) \rightarrow V(\vec{F})$ of $\vec{H}$ in $\vec{F}$ is an isometric copy if $d_{H}(x, y)=d_{F}(f(x), f(y))$ for every $x, y \in V(\vec{H})$. Note that the distance is taken with respect to the underlying undirected graphs.

Given an oriented graph $\vec{H}$ and a graph $G$, we write $G \stackrel{\text { iso }}{\longrightarrow} \vec{H}$ if every orientation of $G$ has an isometric oriented copy of $\vec{H}$. The isometric oriented Ramsey number $\vec{R}_{\text {iso }}(\vec{H})$ is defined as

$$
\vec{R}_{\text {iso }}(\vec{H}):=\inf \{n \in \mathbb{N}: \text { there exists a graph } G \text { of order } n \text { such that } G \stackrel{\text { iso }}{\longrightarrow} \vec{H}\} .
$$

The following result states that the isometric oriented Ramsey number of acyclic oriented graphs is always finite. 
Theorem 6 ([Banakh et al. 2017], Theorem 2.1). For every acyclic oriented graph $\vec{H}$, the isometric oriented Ramsey number $\vec{R}_{\text {iso }}(\vec{H})$ is finite.

The problem of estimating $\vec{R}_{\text {iso }}(\vec{H})$ for acyclic oriented graphs $\vec{H}$ first appeared in Banakh, Idzik, Pikhurko, Protasov and Pszczoła [Banakh et al. 2017], where an upper bound for the isometric Ramsey number of oriented trees is given. Our work gives an upper bound on $\vec{R}_{\text {iso }}(\vec{H})$ when $\vec{H}$ is an acyclic orientation of the cycle on $k$ vertices $C_{k}$. In particular, we prove the following theorem.

Theorem 7. There exists a positive constant $c$ such that the following holds. Let $\vec{H}$ be an acyclic orientation of $C_{k}$ and set $R:=\vec{R}(\vec{H})$. Then

$$
\vec{R}_{\text {iso }}(\vec{H}) \leqslant c k^{12 k^{3}} R^{8 k^{2}} \text {. }
$$

Our approach to prove Theorem 7 employs the same techniques we used to prove Theorem 5. It goes as follows. We consider the random graph $G(n, p)$ and, imitating the proof of Theorem 5, we prove that, with positive probability, we have $G(n, p) \stackrel{\text { iso }}{\longrightarrow} \vec{H}$ for a number $n$ that satisfies (1) and a suitable choice of $p$. In order to show that $G(n, p) \stackrel{\text { iso }}{\longrightarrow} \vec{H}$, we prove that the graph $G(n, p)$ has girth at least $k$ and satisfies $G(n, p) \rightarrow \vec{H}$, which implies $G(n, p) \stackrel{\text { iso }}{\longrightarrow} \vec{H}$. Once again, the proof makes use of the hypergraph container lemma.

\section{References}

Balko, M., Cibulka, J., Král, K., and Kynčl, J. (2015). Ramsey numbers of ordered graphs. Electronic Notes in Discrete Mathematics, 49:419 - 424. The Eight European Conference on Combinatorics, Graph Theory and Applications, EuroComb 2015.

Balogh, J., Morris, R., and Samotij, W. (2015). Independent sets in hypergraphs. J. Amer. Math. Soc., 28(3):669-709.

Banakh, T., Idzik, A., Pikhurko, O., Protasov, I., and Pszczoła, K. (2017). Isometric copies of directed trees in orientations of graphs.

Conlon, D., Dellamonica, Jr., D., La Fleur, S., Rödl, V., and Schacht, M. (2016). A note on induced Ramsey numbers. arXiv e-prints.

Conlon, D., Fox, J., Lee, C., and Sudakov, B. (2017). Ordered Ramsey numbers. J. Combin. Theory Ser. B, 122:353-383.

Erdős, P. and Moser, L. (1964). On the representation of directed graphs as unions of orderings. Magyar Tud. Akad. Mat. Kutató Int. Közl., 9:125-132.

Hàn, H., Retter, T., Rödl, V., and Schacht, M. (2016). Ramsey-type numbers involving graphs and hypergraphs with large girth.

Nenadov, R. and Steger, A. (2016). A short proof of the random Ramsey theorem. Combin. Probab. Comput., 25(1):130-144.

Rödl, V. and Ruciński, A. (1995). Threshold functions for Ramsey properties. J. Amer. Math. Soc., 8(4):917-942.

Rödl, V., Ruciński, A., and Schacht, M. (2016). An exponential-type upper bound for folkman numbers. Combinatorica.

Saxton, D. and Thomason, A. (2015). Hypergraph containers. Invent. Math., 201(3):925992. 